Pacific Journal of Mathematics

ON WEIGHTED POLYNOMIAL APPROXIMATION OF ENTIRE

r unctions 


\title{
ON WEIGHTED POLYNOMIAL APPROXIMATION OF ENTIRE FUNCTIONS
}

\author{
B. A. TAYLOR
}

An existence theorem for the $\bar{\partial}$ operator is used here to prove some results on weighted approximation of entire functions. Theorem 2 shows that if $\phi$ is a convex function on $\mathbb{B}^{n}=R^{2 n}$ such that the Hilbert space of all entire functions $f$ with $\int|f|^{2} e^{-\phi} d \lambda<+\infty$ ( $d \lambda$ Lebesgue measure) contains the polynomials, then the polynomials are dense in this Hilbert space. Two approximation theorems are also given which are related to the theory of quasi-analytic functions.

The method used here is the analogue for $\mathbb{C}^{n}$ of the method used by Hörmander in [7] (see also [6]) to prove approximation theorems for analytic functions in domains of holomorphy. We apply an existence theorem for the $\bar{\partial}$ operator, Theorem 4.4.1 of [7], to prove our Theorem 1, which gives essentially a modification of the results proved in Lemma 4.3.1 and Theorem 4.4.4 of [7]. Our proof is somewhat simpler than the corresponding proofs in [7] because we are working on $5^{n}$ rather than an arbitrary domain of holomorphy, which makes several technical details easier. The rest of the paper then deals with applications of Theorem 1 to weighted approximation of entire functions.

We point out that most of the results proved in this paper can be proved by other methods. For example, Theorems 2 and 5 can be deduced from results in [3]. However, the theorems in this paper are much simpler than the corresponding results of [3]. The methods used here also demonstrate that Hörmander's $L^{2}$ estimates for the $\bar{\partial}$ operator are non-trivial even in one variable, as has already been pointed out by Kiselman [10].

1. Application of the $\bar{\partial}$ existence theorem. We recall briefly some of the results of Hörmander as presented in [7]. Throughout the following $\phi$ denotes a plurisubharmonic function on $\mathbb{\complement}^{n}=\sqrt{ } x \cdots x \sqrt{ }$ ( $n$ times, $\mathbb{E}=$ complex numbers $)$, and $L^{2}(\phi)\left(=L^{2}\left(\mathbb{C}^{n}, \phi\right)\right.$ ) denotes the Hilbert space of functions on $\mathbb{C}^{n}$ which are square integrable with respect to the measure $e^{-\phi} d \lambda$, where $d \lambda$ is the Lebesgue measure. Similarly, $L_{p, q}^{2}(\phi)$ is the space of differential forms of type $(p, q)$ with coefficients from $L^{2}(\phi)$. The collection of all entire functions $f \in L^{2}(\phi)$ is denoted $\mathfrak{U}(\phi)$. A function $f \in L^{2}(\phi)$ is $\mathrm{n} \mathfrak{U}(\phi)$ if and only if $\bar{\partial} f=$ $\sum_{j=1}^{n}\left(\partial f / \partial \bar{z}_{j}\right) d \bar{z}_{j}$ is the zero $(0,1)$ form (with derivatives taken in the sense of distributions).

We shall use a special case of Theorem 4.4.1 of [7]. 
THEOREM. Let $\phi$ be a strictly plurisubharmonic function of class $C^{2}$ such that

$$
\sum_{j, k=1}^{n}\left(\partial^{2} \phi / \partial z_{j} \partial \bar{z}_{k}\right) w_{j} \bar{w}_{k} \geqq c \sum_{j=1}^{n}\left|w_{j}\right|^{2}
$$

where $c(z)$ is a positive continuous function and $z=\left(z_{1}, \cdots, z_{n}\right), w=$ $\left(w_{1}, \cdots, w_{n}\right) \in \mathbb{\mho}^{n}$. For every $g=\sum_{j=1}^{n} g_{i} d \bar{z}_{j} \in L_{0,1}^{2}(\phi)$ such that $\bar{\partial} g=0$ and

$$
\int|g|^{2} e^{-\phi} / c d \lambda<+\infty\left(|g|^{2}=\sum_{j=1}^{n}\left|g_{j}\right|^{2}\right),
$$

there exists $u \in L^{2}(\phi)$ such that $\bar{\partial} u=g$ and

$$
\int|u|^{2} e^{-\phi} d \lambda \leqq \int|g|^{2} e^{-\phi} / c d \lambda
$$

Actually, we need a slightly stronger version of the theorem in which the hypothesis that $\phi \in C^{2}$ is relaxed. The weight functions we wish to apply the theorem to are of the form $\phi(z)+\log \left(1+|z|^{2}\right)$ $\left(|z|^{2}=\sum_{j=1}^{n}\left|z_{j}\right|^{2}\right.$ for $\left.z=\left(z_{1}, \cdots, z_{n}\right) \in \mathbb{C}^{n}\right)$, where $\phi$ does not belong to the class $C^{2}$. However, as pointed out by Hörmander in [8], the theorem is easily extended to this case by the same technique used in the proof of Theorem 4.4.2 of [7]. Also from this proof, we see that for weight functions of this form, we can take $c(z)=\left(1+|z|^{2}\right)^{-2}$.

THEOREM 1. Let $\phi_{1} \leqq \phi_{2} \leqq \phi_{3} \leqq \cdots$ be plurisubharmonic functions on $\mathbb{( 5}^{n}$, let $\phi=\lim _{j \rightarrow+\infty} \phi_{j}$, and suppose that $\int_{K} \exp \left(-\phi_{1}\right) d \lambda<+\infty$ for every compact set $K$. Then the closure of $\mathbf{U}_{j=1}^{\infty} \mathfrak{2}\left(\phi_{j}+\log \left(1+|z|^{2}\right)\right)$ in the Hilbert space $L^{2}\left(\phi+\log \left(1+|z|^{2}\right)\right)$ contains $\mathfrak{A}(\phi)$.

Proof. Let $\alpha_{1}, \alpha_{2}, \cdots$ be a sequence of $C^{\infty}$ functions with compact support such that $\alpha_{n}(z)=1$ for $|z| \leqq n, 0 \leqq \alpha_{n} \leqq 1$, and

$$
\sum_{1}^{n}\left|\frac{\partial \alpha_{n}}{\partial \bar{z}_{j}}\right|^{2} \leqq K\left(1+|z|^{2}\right)^{-1}
$$

(For example, let $\chi \in C^{\infty}$ have compact support, $0 \leqq \chi \leqq 1$ and $\chi(z)=1$ for $|z| \leqq 1$. Then put $\alpha_{n}(z)=\chi(z / n)$.) Let $f \in \mathfrak{A}(\phi)$. Then $f \alpha_{n}$ is a good approximation to $f$, but it is not analytic, so we modify it as usual. That is, put $g_{n}=\bar{\partial}\left(f \alpha_{n}\right)=f \bar{\partial} \alpha_{n}$. We will use the above existence theorem to solve the equation $\bar{\partial} u_{n}=g_{n}$ so that the analytic function $a_{n}=f \alpha_{n}-u_{n}$ belongs to $\mathbf{U}_{j} \mathfrak{A}\left(\dot{\phi}_{j}+\log \left(1+|z|^{2}\right)\right)$ and converges to $f$ in the Hilbert space $\mathfrak{A}\left(\phi+\log \left(1+|z|^{2}\right)\right)$.

Let $\psi_{j}(z)=\phi_{j}(z)+\log \left(1+|z|^{2}\right)$, and let $\psi$ be defined in the same way with $\phi_{j}$ replaced by $\phi$. Then define 


$$
I(n, j)=\int\left|g_{n}\right|^{2}\left(1+|z|^{2}\right)^{2} e^{-\psi j} d \lambda=\int\left|g_{n}\right|^{2}\left(1+|z|^{2}\right) e^{-\phi_{j}} d \lambda,
$$

and let $I(n)$ be defined in the same way with $\psi_{j}$ replaced by $\psi$. Now $I(n)=\int|f|^{2}\left|\bar{\partial} \alpha_{n}\right|^{2}\left(1+|z|^{2}\right) e^{-\phi} d \lambda \leqq K \int_{|z| \geqq n}|f|^{2} e^{-\phi} d \lambda=\varepsilon_{n}$ and $\varepsilon_{n} \rightarrow 0$ as $n \rightarrow \infty$, since $\int|f|^{2} e^{-\phi} d \lambda<+\infty$. Moreover, $g_{n}$ has compact support and the $\phi_{j}$ increase monotonely to $\phi$. Therefore, $\lim _{j \rightarrow+\infty} I(n, j)=I(n)$ by the dominated convergence theorem, so there is an integer $j=j(n)$ such that $I(n, j(n))<2 \varepsilon_{n}$. Then by the above theorem (and remarks) applied with the weight function $\left(\psi_{j(n)}\right)$ there exists $u_{n}$ such that $\bar{\partial} u_{n}=g_{n}$ and

$$
\int\left|u_{n}\right|^{2} e^{-\psi \psi_{j}} d \lambda<2 \varepsilon_{n} \quad \quad(j=j(n)) .
$$

If $a_{n}=f \alpha_{n}-u_{n}$, then $a_{n} \in \bigcup_{j} \mathfrak{R}\left(\psi_{j}\right)$. Moreover,

$$
\int\left|u_{n}\right|^{2} e^{-\psi} d \lambda \leqq \int\left|u_{n}\right|^{2} e^{-\psi j} d \lambda \leqq 2 \varepsilon_{n} \quad(j=j(n))
$$

so $v_{n} \rightarrow 0$ in $L^{2}(\psi)$. Also $f \alpha_{n} \rightarrow f$ in $L^{2}(\psi)$, so that $a_{n} \rightarrow f$ as asserted.

REMARKS. 1. It is not necessarily true that $\mathscr{H}(\phi)$ is dense in $\mathfrak{U}\left(\phi+\log \left(1+|z|^{2}\right)\right)$. For example, take, in one variable

$$
\phi(z)=\frac{3}{2} \log \left(1+|z|^{2}\right)
$$

so that $\mathfrak{A}(\phi)$ is the constants and $\mathfrak{U}\left(\phi+\log \left(1+|z|^{2}\right)\right)$ is the polynomials of degree $\leqq 1$. However, we know of no nontrivial example in which $\mathfrak{U}(\phi)$ is not dense in $\mathfrak{X}\left(\dot{\phi}+\log \left(1+|z|^{2}\right)\right)$. Also, we know of no example in which $\bigcup_{j} \mathfrak{A}\left(\phi_{j}\right)$ fails to be dense in the Hilbert space $\mathfrak{A}(\phi)$.

2. Note that to prove $\mathfrak{X}(\phi)$ is dense in $\mathfrak{U}\left(\phi+\log \left(1+|z|^{2}\right)\right)$, it suffices to prove that the reproducing kernel $K(z, w)$ for this latter Hilbert space belongs to $\mathfrak{A}(\phi)$, (as a function of one variable with the other held fixed). This is the case in every nontrivial example we know. However, we have been unable to prove this estimate for any reasonably general class of weight functions.

3. As is well known, we note that questions of polynomial approximation are trivial when $\phi(z)=\phi(|z|)$ is a function only of the distance from the origin.

Corollary. $(n=1)$ Suppose $\int e^{-\phi_{1}} d \lambda<+\infty$, and let 


$$
S \mathfrak{U}(\phi)=\left\{f \in \mathfrak{Y}(\phi):(1+|z|) f \in L^{2}(\phi)\right\} \text {. }
$$

Then the closure of $\bigcup_{j} \mathfrak{X}\left(\phi_{j}\right)$ in $\mathfrak{U}(\phi)$ contains $S \mathfrak{X}(\phi)$.

Proof. If $f \in S \mathfrak{U}(\phi)$, then $z f \in \mathfrak{U}(\phi)$ and then by Theorem 1 there are functions $a_{n}$ is $\mathbf{U}_{j} \mathfrak{A}\left(\phi_{j}+\log \left(1+|z|^{2}\right)\right)$ which converge to $z f$ in $\mathfrak{U}\left(\phi+\log \left(1+|z|^{2}\right)\right)$. It is then easy to check that the functions $b_{n}(z)=\left(a_{n}(z)-a_{n}(0)\right) / z$ belong to $\bigcup_{j} \mathfrak{Y}\left(\dot{\phi}_{j}\right)$ and converge to $f$ in $\mathfrak{U}(\phi)$.

2. Convex weight functions. In case the plurisubharmonic weight function $\phi$ is a convex function on $\mathbb{5}^{n}=R^{2 n}$, we can completely settle the question of when the polynomials are dense in $\mathfrak{A}(\phi)$. Recall that for a convex function $\phi$ on $R^{2 n}=\sqrt{5}^{n}$, the conjugate convex function to $\phi, \phi^{*}$, is defined by

$$
\phi^{*}(w)=\sup \left\{\operatorname{Re}\langle z, w\rangle-\phi(z): z \in \complement^{n}\right\},
$$

where

$$
\langle z, w\rangle=\sum_{1}^{n} z_{j} \bar{w}_{j}, \quad z, w \in \mathfrak{C}^{n} .
$$

THEOREM 2. Let $\phi$ be convex on $\mathbb{C}^{n}$.

(i) If $1 \in \mathfrak{A}(\phi)$, then $\phi^{*}(w)$ is finite on a neighborhood of the origin in $\mathbb{E}^{n}$.

(ii) If $\phi^{*}$ is finite on a neighborhood of the origin in $\mathbb{C}^{n}$, then the polynomials are dense in $\mathfrak{U}(\phi)$.

The proof of (ii) of Theorem 2 is essentially an application of Theorem 1, where the $\phi_{j}$ are finite maximums of suitable tangent planes to the surface $y=\phi(z)$, and part (i) is just a fact about convex functions. Before proving Theorem 2, we shall collect some elementary facts about convex functions which will be needed in the proof.

Definition. (a) $\mathscr{L}(\phi)=\left\{\ell(z): \ell(z) \leqq \phi(z)\right.$ for all $\left.z \in \mathbb{C}^{n}\right\}$, where $\measuredangle(z)=\operatorname{Re}\langle z, w\rangle+A$ for some $w \in \mathbb{C}^{n}$ and some constant $A$.

(b) $F(\phi)=\left\{w \in \mathfrak{C}^{n}: \phi^{*}(w)<+\infty\right\}$.

(c) $F^{0}(\phi)=$ interior of $F(\phi)$.

Note that $F(\phi), F^{0}(\phi)$ are convex, though possibly empty.

Proposition 1. If $K$ is a compact subset of $F^{0}(\phi)$ of distance greater than $\delta>0$ from the complement of $F^{0}(\phi)$, then

$$
\dot{\phi}(z) \geqq h(z, K)+\delta|z|-A
$$

where 


$$
h(z, K)=\operatorname{Sup}\{\operatorname{Re}\langle z, w\rangle: w \in K\}
$$

is the support function of $K$ and $A=A(K, \delta)$ is a constant.

Proof. Let $K^{\prime}$ denote the set of all points of $\mathbb{C}^{n}$ which are of distance at most $\delta$ from $K$. Then $K^{\prime}$ is a compact subset of $F^{0}(\dot{\phi})$. Now $\phi^{*}$ is continuous on $F^{0}(\phi)$ (see, e.g., [4]), and thus bounded on $K^{\prime}$, say $A=\sup \left\{\phi^{*}(w): w \in K^{\prime}\right\}$. Let $z \in \mathbb{C}^{n}$. Choose $u \in \mathbb{C}^{n},|u|=\delta$, such that $\langle z, u\rangle=\delta|z|$. For each $w \in K$, the vector $w+u \in K^{\prime}$. Thus $A \geqq \dot{\phi}^{*}(w+u) \geqq \operatorname{Re}\langle z, w+u\rangle-\phi(z)=\operatorname{Re}\langle z, w\rangle-\phi(z)+\delta|z|$. This holds for all $w \in K$, so $A \geqq h(z, K)-\dot{\phi}(z)+\delta|z|$, which is equivalent to the desired inequality.

Proposition 2. Let $F(\phi)$ be as above. If $F^{0}(\phi)$ is empty, then $F$ is contained in a (real) $2 n-1$ dimensional hyperplane. Moreover, $\phi$ is constant on lines perpendicular to this hyperplane.

Proof. The set $F$ where $\phi^{*}$ is finite is clearly convex, since

$$
\phi^{*}\left(\alpha w_{1}+(1-\alpha) w_{2}\right) \leqq \alpha \phi^{*}\left(w_{1}\right)+(1-\alpha) \phi^{*}\left(w_{2}\right)
$$

for $0<\alpha<1$, and any convex set in $R^{2 n}=\mathbb{C}^{n}$ with empty interior is contained in some (not necessarily unique) hyperplane. Suppose such a hyperplane is

$$
H=\left\{w \in \complement^{n}: \operatorname{Re}\left\langle z^{(0)}, w\right\rangle+A=0\right\} \quad \text { where } \quad z^{(0)} \in \Im^{n}, A \in R .
$$

Now, $\phi(z)=\sup \{\ell(z): \ell \in \mathscr{L}(\phi)\}$ (see, e.g., [4], or apply the HahnBanach theorem). Let $\ell \in \mathscr{L}(\phi)$. Then $\ell(z)=\operatorname{Re}\langle z, w\rangle+c$ for some constant $c$ and some $w \in \mathbb{C}^{n}$, and

$$
\operatorname{Re}\langle z, w\rangle+c \leqq \phi(z) .
$$

This implies that $\phi^{*}(w)<+\infty$, hence that $w \in H$. Therefore, $\ell(z)$ is constant on all one (real) dimensional lines orthogonal to $H$, and $\phi(z)$, as the upper envelope of all the $\ell \in \mathscr{L}(\dot{\phi})$, is also constant on such lines.

Proposition 3. Suppose that $F^{0}(\phi)$ contains the origin. Then there is a compact subset $K$ of $\mathbb{S}^{n}$ and a number $\varepsilon>0$ such that

$$
\dot{\phi}(\theta z)-\dot{\phi}(z) \geqq \varepsilon(\theta-1)|z|
$$

for all $\theta>1$ and all $z \notin K$.

Proof. We will prove this by obtaining a lower bound on $(\partial / \partial t) \dot{\phi}(t z)$ for large real numbers $t$. Let $\varepsilon>0$ be such that the closed ball of 
radius $4 \varepsilon$, and center at the origin is contained in $F^{\circ}(\phi)$. If $K$ denotes the closed ball of radius $2 \varepsilon$ and center at the origin, then $h(z, K)=$ $2 \varepsilon|z|$ so from Proposition 1,

$$
\dot{\phi}(z) \geqq 2 \varepsilon|z|-A \text {. }
$$

Consider for $z \in \sqrt{ }^{n},|z|=1$, and for $t \in R$, the convex function of $t$,

$$
g(t)=g_{z}(t)=\phi(t z) \text {. }
$$

Let $d g / d t$ denote the left hand derivative of $g(t)$. Since the difference quotients of a convex function are nondecreasing,

$$
\left.\frac{d g}{d t}=\sup \left\{\left(g^{\prime}(t+\Delta t)-g^{\prime} t\right)\right) / \Delta t: \Delta t<0\right\} \text {. }
$$

For large positive $t, g(t) \geqq 2 \varepsilon t|z|-A$ and consequently $d g / d t>\varepsilon$. Define, for $r>0, E(r)=\left\{z \in \mathbb{C}^{n},|z|=1: d g / d t(r)>\varepsilon\right\}$. What we have just proved above is that $\bigcup_{r>0} E(r)=\left\{z \in \mathfrak{\Im}^{n}:|z|=1\right\}$. But $d g / d t$, as a function of $z$, is lower semicontinuous, because it is the upper envelope of a family of continuous functions. This implies that each $E(r)$ is open. Since the sets $E(r)$ increase with increasing $r$, it follows from the compactness of $\left\{z \in \mathbb{C}^{n}:|z|=1\right\}$ that for some $r_{0}>0, E\left(r_{0}\right)=$ $\left\{z \in \mathbb{S}^{n}:|z|=1\right\}$. Then if $|z| \geqq r_{0}$, and $\theta>1$,

$$
\begin{aligned}
\phi(\theta z)-\phi(z) & =\phi\left(\theta|z|\left(z /\left|z_{i}^{\prime}\right|\right)\right)-\phi(|z|(z /|z|)) \\
& =\int_{|z|}^{\theta|z|} \frac{d g}{d t} d t>\varepsilon(\theta|z|-|z|)=\varepsilon(\theta-1)|z|,
\end{aligned}
$$

as asserted.

Proof of Theorem 2 (i). Assume, by way of contradiction, that $\phi^{*}(w)$ is not finite on a neighborhood of the origin in $\mathbb{C}^{n}$. Then, since $F(\phi)$ is convex, there is a $z \in \mathbb{C}^{n}$ such that $\operatorname{Re}\langle z, w\rangle \leqq 0$ for all $w \in F(\phi)$. After perhaps a linear change of variable, we may suppose that $z=$ $(1,0,0, \cdots, 0)$, so that $\operatorname{Re} w_{1} \leqq 0$ for all $w=\left(w_{1}, \cdots, w_{n}\right) \in F(\phi)$. But, $\phi(z)$ is the upper envelope of the functions $\ell(z) \in \mathscr{L}(\phi)$, and each such $\iota(z)$ has the form $\ell(z)=\operatorname{Re}\langle z, w\rangle+c$ where $w \in F(\phi)$. Therefore, each $\ell(z)$ is nonincreasing in the variable $\operatorname{Re} z_{1}$, and so the same is true of $\phi(z)$. This clearly implies that $\int e^{-\phi} d \lambda=+\infty$, which contradicts the assumption that $1 \in \mathfrak{Y}(\phi)$. Thus, (i) is proved.

Proof of Theorem 2 (ii). If $F^{\circ}(\phi)$ contains the origin, then we may choose $\varepsilon>0$ such that $\mathscr{O}=\{w: 2|w| \leqq \varepsilon\}$ is contained in $F^{\circ}(\phi)$. If $f(z)=\exp \langle z, w\rangle$, with $w \in \mathcal{O}$, then it is easy to verify that the power series of $f$ converges to $f$ in $\mathfrak{U}(\dot{\phi})$. Therefore, (ii) will follow from 
assertions (a)-(d) below. The proofs of (a), (b), and (d) are routine, and (c) follows from Theorem 1.

(a) Let $\mathcal{O}$ denote an open subset of $F^{0}(\phi)$. Then for each $w \in \mathcal{O}$, $\exp \left(\frac{1}{2}\langle z, w\rangle\right) \in \mathfrak{A}(\phi)$ and the closure of the linear span of all such exponential functions contains $\exp \left(\frac{1}{2}\langle z, w\rangle\right)$ for all $w \in F^{0}(\phi)$.

(b) The closure of the linear span of the exponentials $\exp \left(\frac{1}{2}\langle z, w\rangle\right)$, with $w \in F^{0}(\phi)$ contains all entire functions of exponential type $f(z)$ of the form

$$
f(z)=\int \exp (\langle z, w\rangle) d \mu(w)
$$

where $\mu$ is a Borel measure of compact support, whose support is contained in $\left\{\frac{1}{2} w: w \in F^{0}(\phi)\right\}=\frac{1}{2} F^{0}(\phi)$.

(c) The closure in $\mathfrak{U}(\phi)$ of the entire functions of exponential type described in (b) contains all $f \in \mathfrak{U}(\phi)$ such that $f(\theta z) \in \mathfrak{N}(\phi)$ for some real number $\theta>1$.

(d) If $f \in \mathfrak{U}(\dot{\phi})$ and $r<1$, then $f(r z) \in \mathfrak{U}(\dot{\phi})$ and $\lim _{r \rightarrow 1^{-}} f(r z)=f(z)$ in $\mathfrak{Y}(\phi)$.

We first prove (a) and (b). Let $g \in \mathfrak{U}(\dot{\phi})$ be orthogonal to all $\exp \left(\frac{1}{2}\langle z, w\rangle\right)$, with $w \in \mathcal{O}$. The estimate of Proposition 1 allows us to prove easily that

$$
G(w)=\int \overline{g(z)} \exp \left(\frac{1}{2}\langle z, w\rangle\right) \exp (-\dot{\phi}(z)) d \lambda(z)
$$

is conjugate analytic for $w \in F^{0}(\phi)$. By hypothesis, $G$ vanishes for $w \in \mathcal{O}$ so $G$ vanishes on $F^{0}(\dot{\phi})$ since $F^{0}(\phi)$ is connected (even convex). Part (a) then follows by the Hahn-Banach theorem. The proof of (b) is essentially the same. If $f(z)$ is of the form given there, then

$$
\int \overline{g(z)} f(z) \exp (-\dot{\phi}(z)) d \lambda(z)=\int G(w) d \mu(w)=0,
$$

the interchange in order of integration being justified by Fubini's theorem and the inequality of Proposition 1.

We will use Theorem 1 to prove (c). Let $r<1$ and $\psi(z)=\psi_{r}(z)=$ $\phi(r z)$. We want to choose $\dot{\phi}_{1} \leqq \phi_{2} \leqq \cdots$ with $\sup _{j} \phi_{j}=\psi$ in such a way that $\mathbf{U}_{j} \mathfrak{Y}\left(\dot{\phi}_{j}+\log \left(1+|z|^{2}\right)\right)$ is a subset of the class of functions described in (b). To do this, let $\iota_{1}(z), \iota_{2}(z), \ldots$ be a sequence of functions in $\mathscr{C}(\psi)$ such that $\sup \left\{\ell_{j}(z): j=1,2, \cdots\right\}=\psi(z)$, and then define $\phi_{j}(z)=\max \left\{\ell_{1}(z), \cdots, \ell_{j}(z)\right\}$. (To see that such functions $\ell_{j}$ exist, note that each $\ell \in \mathscr{L}(\psi)$ is of the form $\ell(z)=\operatorname{Re}\langle z, w\rangle-c$ for some $c \in R$, $w \in \mathfrak{C}^{n}$. Set $B=\left\{(w, c) \in \mathbb{C}^{n} \times R: \ell(z) \in \mathscr{L}(\psi)\right\}$. Then $B$ has a countable dense subset $\left(w_{n}, c_{n}\right)$ and

$$
{ }_{n}^{\prime}(z)=\operatorname{Re}\left\langle z, w_{n}\right\rangle-c_{n}
$$


is a suitable sequence.) It then follows from Theorem 1 that the closure of $\bigcup_{j} \mathfrak{A}\left(\phi_{j}+\log \left(1+|z|^{2}\right)\right)$ in $\mathfrak{U}\left(\psi+\log \left(1+|z|^{2}\right)\right)$ contains $\mathfrak{X}(\psi)$. By Proposition $3, \psi(z)+\log \left(1+|z|^{2}\right) \leqq \phi(z)$, except possibly on a compact subset of $\mathbb{C}^{n}$ and, consequently, convergence in

$$
\mathfrak{U}\left(\psi+\log \left(1+|\boldsymbol{z}|^{2}\right)\right)
$$

implies convergence in $\mathfrak{U}(\phi)$.

To complete the proof of $(c)$, it remains to show that each

$$
f \in \mathfrak{U}\left(\phi_{j}+\log \left(1+|z|^{2}\right)\right)
$$

is of the form prescribed in (b). Let $\psi_{j}=\phi_{j}+\log \left(1+|z|^{2}\right)$. By construction of the $\phi_{n}$, there are points $w_{1}, \cdots, w_{j} \in \mathbb{C}^{n}$ and constants $c_{1}, \cdots, c_{j}$ such that $\phi_{j}$ is the maximum of $\operatorname{Re}\left\langle z, w_{k}\right\rangle-c_{k}, k=1,2, \cdots, j$. In particular, it follows easily that

$$
\widetilde{\psi}_{j}(z)=\sup \left\{\psi_{j}(z+u):|u| \leqq 1\right\} \leqq \psi_{j}(z)+\text { Const. }
$$

But for $f \in \mathfrak{U}\left(\psi_{j}\right)$ we have, since $f$ is analytic

$$
f(z)=\frac{1}{k_{n}} \int_{|u| \leqq 1} f(z+u) d \lambda(u)
$$

where $k_{n}$ is the volume of the unit ball in $\mathbb{C}^{n}$. Thus

$$
\begin{aligned}
|f(z)| \leqq & \frac{1}{k_{n}} \int_{|u| \leqq 1}|f(z+u)| \exp \left(-\frac{1}{2} \psi_{j}(z+u)+\frac{1}{2} \psi_{j}(z+u)\right) d \lambda(u) \\
& \leqq \frac{1}{k_{n}} \exp \left(\frac{1}{2} \widetilde{\psi}_{j}(z)\right) \int_{|u| \leqq 1}|f(z+u)| \exp \left(-\frac{1}{2} \psi_{j}(z+u)\right) d \lambda(u) .
\end{aligned}
$$

Applying Schwarz's inequality to this last integral, and then extending the domain of integration to all of $\mathbb{C}^{n}$, we find

$$
|f(z)| \leqq\|f\| \cdot \exp \left(\frac{1}{2} \tilde{\psi}_{j}(z)\right),
$$

where $|f|$ is the norm in $\mathfrak{A}\left(\psi_{j}\right)$. Therefore,

$$
|f(z)| \leqq \text { Const. }\|f\| \exp \left(\frac{1}{2} \psi_{j}(z)\right) \text {. }
$$

Now each $\ell_{j}(z) \leqq \psi(z)=\phi(r z)$ where $r<1$, so each $w_{j}$ occurring in the representation of $\ell_{j}(z)$ necessarily belongs to $F^{0}(\phi)$. Also,

$$
\log \left(1+|z|^{2}\right)=0(\varepsilon|z|)
$$

for every $\varepsilon>0$. Thus, $\psi_{j}(z) \leqq h(z, K)+0(1)$ for some compact subset $K\left(=K_{j}\right)$ of $F^{0}(\phi)$. Thus,

$$
|f(z)|=0\left(\exp \left(\frac{1}{2} h(z, K)\right)\right)=0\left(\exp \left(h\left(z, \frac{1}{2} K\right)\right)\right) .
$$

It then follows that $f(z)$ is of the form in part (b) (see, e.g., Martineau 
$[12$, p. 150], or Hörmander [7, p. 98], or for the case $n=1,[1$, p. 74]). This completes the proof of (c).

Lastly, we prove (d). Let $f \in \mathscr{Y}(\dot{\phi})$. We will use the fact that if $f_{j} \rightarrow f$ pointwise and $\left\|f_{j}\right\| \rightarrow\|f\|$, then $f_{j} \rightarrow f$ in $\mathfrak{N}(\phi)$ (see, e.g., [5, p. 209]). Clearly $\lim _{r \rightarrow 1}-f(r z)=f(z)$ pointwise. Also

$$
|f(r z)|^{2}=\int|f(r z)|^{2} \exp (-\dot{\phi}(z)) d \lambda(z)=\frac{1}{r^{2 n}} \int|f(z)|^{2} \exp (-\dot{\phi}(z / r)) d \lambda(z) .
$$

Outside the compact set of Proposition 3, $\dot{\phi}(z / r)$ decreases monotonely to $\phi(z)$, while on the compact set, $\phi(z / r)$ converges uniformly to $\dot{\phi}(z)$. Thus, $\|f(r z)\|_{i}^{2}$ converges to

$$
\int|f(z)|^{2} \exp (-\phi(z)) d \lambda(z)=\|f\|^{2} .
$$

This completes the proof of Theorem 2, (ii).

When $\phi^{*}$ is just finite on an open set, we can make the followingadjustment of Theorem 2 .

THEOREM 3. Let $\dot{\phi}$ be conve: on $\Gamma^{n}$, and let $F^{0}(\dot{\rho})$ denote the interior of the set on which $\dot{\phi}^{*}(w)<+\infty$.

(i) If $f(z)=\exp (\langle z, w\rangle) \in \mathfrak{Q}(\dot{\phi})$, then $2 w \in F^{0}(\dot{\phi})$, and

(ii) If $F^{\circ}(\phi)$ is not empty, then the collection of functions $f(z)=$ $\exp (\langle z, w\rangle)$ with $2 w \in F^{0}(\dot{\phi})$ have dense linear span in $\mathfrak{Y}(\dot{\phi})$.

Proof. If $w_{0} \in \mathbb{C}^{n}$ and $\dot{\phi}_{0}(z)=\dot{\phi}(z)-\operatorname{Re}\left\langle z, w_{0}\right\rangle$, then the mapping $f(z) \rightarrow f(z) \cdot \exp \left(\frac{1}{2}\left\langle z, w_{>}\right\rangle\right)$is an isometric isomorphism of $\mathfrak{Y}\left(\dot{\phi}_{0}\right)$ onto $\mathfrak{Y}(\dot{\phi})$ which carries exponential functions onto exponential functions. Also, the set on which $\dot{\phi}_{0}^{*}$ is finite is the translate by $-w_{0}$ of the set on which $\phi^{*}$ is finite. The above theorem then follows from Theorem 2, or more correctly, from assertions (a)-(d) of the proof of Theorem 2 applied to the space $\mathfrak{R}\left(\phi_{0}\right)$ with $\dot{\phi}_{0}$ defined using an element $w_{0} \in F^{0}(\dot{\rho})$.

We can also prove Theorems 2 and 3 for norms other than the $L^{2}$ norm.

Definition. (a) $\mathfrak{Y i n}_{\mathrm{w}}^{p}(\dot{\phi})=\left\{f\right.$ entire: $\left.\|f\|_{p}=\left(\int|f|^{p} e^{-\phi} d \lambda\right)^{1 / p}<+\infty\right\}$

(b) $\mathfrak{i}^{\infty}(\dot{\phi})=\left\{f\right.$ entire: $f(z) e^{-\phi(z)} \rightarrow 0$ as $\left.|z| \rightarrow+\infty\right\}$, and $\|f\|_{\infty}=$ $\sup \left\{|f(z)| e^{-\phi(z)}: z \in \mathbb{C}^{n}\right\}$.

THEOREM 4. If $\dot{\rho}$ is conver on $\mathbb{C}^{n}$ and if $\dot{\rho}^{*}$ is finite on a neighborhood of the origin, then the polynomials are dense in the Banach spaces $\mathrm{Y}^{r}(\dot{\rho}), 1 \leqq p \leqq+\infty$. 
Proof. We prove this only for finite $p$, the case $p=\infty$ being essentially the same. As in (d) of the proof of Theorem 2, we have $f(r z) \rightarrow f(z)$ in $\mathfrak{U}^{p}(\phi)$ as $r \rightarrow 1^{-}$. Fix $r<1$ and choose $r<\rho<\rho^{\prime}<$ $\rho^{\prime \prime}<1$. Then exactly as proved in (c) of Theorem 2 ,

$$
|f(r z)| \leqq\|f\|_{p} \exp \left(\frac{1}{p} \tilde{\phi}(r z)\right),
$$

where $\tilde{\phi}(z)=\sup \{\phi(z+u):|u|=1\}$. By Proposition 3,

$$
\tilde{\phi}(r z) \leqq \phi(\rho z)+0(1) \leqq \phi\left(\rho^{\prime} z\right)-\left(\rho^{\prime}-\rho\right)|z|+0(1) .
$$

Hence $f(r z) \in \mathfrak{Y}\left((2 / p) \dot{\phi}\left(\rho^{\prime} z\right)=\mathfrak{I}^{2}\left((2 / p) \phi\left(\rho^{\prime} z\right)\right)\right.$. Apply Theorem 2 to obtain polynomials $p_{j}(z)$ converging to $f(r z)$ in this Hilbert space. Then these polynomials also converge to $f(r z)$ in $\mathfrak{U}^{p}(\dot{\phi})$. For, we have pointwise convergence and the bound, derived as above,

$$
\left|p_{j}(z)\right| \leqq \text { Const. } \exp \left(\frac{1}{p} \tilde{\phi}\left(\rho^{\prime} z\right)\right)
$$

Whence, by Proposition 3,

$$
\tilde{\phi}\left(\rho^{\prime} z\right) \leqq \dot{\phi}\left(\rho^{\prime \prime} z\right)+0(1) \leqq \dot{\phi}(z)-\left(1-\rho^{\prime \prime}\right)|z|+0(1),
$$

and therefore $\int\left|p_{j}(z)-f(r z)\right|^{p} e^{-\phi} d \lambda$ converges to zero by the dominated convergence theorem.

3. Weight functions of the form $v(r)+u(x)$. We study in this section weight function of the form $\phi(z)=v(r)+u(x)$, where

$$
z=\left(z_{1}, \cdots, z_{n}\right), r=\left(\left|z_{1}\right|, \cdots,\left|z_{n}\right|\right),
$$

and $x=\left(\operatorname{Re} z_{1}, \cdots, \operatorname{Re} z_{n}\right)=\left(x_{1}, \cdots, x_{n}\right)$. In order to guarantee that $\phi$ be plurisubharmonic, we assume that the following conditions are satisfied.

(i) $u(x)$ is convex;

(ii) $v(r)$ is a convex function of $\log r$ (i.e., $v\left(e^{r_{1}}, \cdots, e^{r_{n}}\right)$ is convex);

To insure that 织 $(\dot{\phi})$ contains the polynomials, we assume

(iii) $v(r) \geqq A \sum_{i=1}^{n} \log \left(1+r_{i}\right)$ for each $A>0$ and $r_{1}+\cdots+r_{n}$ sufficiently large.

In order that the space $\mathfrak{i}(\phi)$ contains some exponential functions with frequencies near zero, we also assume

(iv) $u(x) \geqq \delta\left(\left|x_{1}\right|+\cdots+\left|x_{n}\right|\right)-C$ for some $\delta>0$ and some constant $C$.

Lastly, we assume a technical condition which is needed in our proof. 
(v) $v\left(r_{1}, \cdots, r_{n}\right)$ is nondecreasing in each variable $r_{i}$.

From (iv) it follows that $u^{*}(t)=\sup _{x}\left(t_{1} x_{1}+\cdots+t_{n} x_{n}-u(x)\right)$ where $x=\left(x_{1}, \cdots, x_{n}\right), x_{i}$ real, is finite on some neighborhood of the origin in $R^{n}$.

DEFINITION. $E(u)=\left\{\exp \left(\left\langle z, t_{0}\right\rangle\right): u^{*}(t)\right.$ is finite on a neighborhood of $t_{0}$ in $R^{n}$.

The following theorem is useful in proving that certain spaces of quasi-analytic functions are analytically uniform [15].

THEOREM 5. If $\phi(z)=v(r)+u(x)$ satisfies (i)-(iv) above, then the linear span of the exponential functions in $E(u)$ is dense in $\mathfrak{U}^{p}(\dot{\phi})$, $1 \leqq p \leqq+\infty$.

Proof. The proof is quite similar to that of Theorems 2 and 4, so we shall only outline the steps for the case $p=2$. The functions $\phi_{j}$ may be chosen as follows. Let $u_{j}(x)$ be an increasing sequence of convex functions such that $u_{j}(x) \leqq u(x), u_{j}(x) \rightarrow u(x)$, and

$$
u_{j}(x)=0\left(\left|x_{1}\right|+\cdots+\left|x_{n}\right|\right)
$$

for each $j>0$. Such a sequence may be constructed as in the proof of $(c)$ of Theorem 2. Let $v_{j}(r)$ be an increasing sequence of convex functions of $\log r$ (as described in (ii) above) such that $v_{j}(r) \leqq v(r)$, $v_{j}(r) \rightarrow v(r)$ and

$$
v_{j}(r)=0\left(\sum_{i=1}^{n} \log \left(1+r_{i}\right)\right) \quad \text { for each } j>0 .
$$

Such a sequence may be constructed by passing to the convex function $v\left(e^{r_{1}}, \cdots, e^{r_{n}}\right)$, and proceeding as before to construct $v_{j}\left(e^{r_{1}}, \cdots e^{r_{n}}\right)$, which thus defines $v_{j}$. Then $\phi_{j}(z)=u_{j}(r)+v_{j}(x)$ is plurisubharmonic and $\phi_{1} \leqq \phi_{2} \leqq \cdots, \phi_{j} \rightarrow \phi$.

Now exactly as in the proof of (d) of Theorem $2, f(r z) \rightarrow f(z)$ as $r \rightarrow 1$ for each $f \in \mathfrak{U}(\phi)$. Therefore,

$$
V=\bigcup_{r<1} \bigcup_{\jmath} \mathfrak{A}\left(\phi_{j}(r z)\right)
$$

is dense in $\mathfrak{X}(\phi)$, by Theorem 1 . Consequently, it suffices to prove that every $f \in V$ is a limit, in $\mathfrak{U}(\phi)$, of linear combinations of the $\exp (\langle z, t\rangle)$. Each $f \in V$ satisfies an estimate of the form

$$
|f(z)| \leqq A\left(1+r_{1}+\cdots+r_{n}\right)^{B} \exp \left(\frac{1}{2} h(x, K)\right)
$$

for some constants $A, B$, where $h(x, K)$ is the support function of some compact subset $K$ of the interior of the set of all $y=\left(y_{1}, \cdots, y_{n}\right), y_{j}$ 
real, such that

$$
u^{*}(y)=\sup _{x}\left(y_{1} x_{1}+\cdots+y_{n} x_{n}-u(x)\right)<+\infty .
$$

Further, each $f$ satisfying (3.1) is the limit in $\mathfrak{U}(\phi)$ of functions of the form

$$
f(z)=\int e^{\langle z, t\rangle} g(t) d \lambda(t)
$$

where $g$ is an $L^{2}$ function with compact support contained in the interior of the set of all $y$ satisfying (3.2). This may be seen for example, by multiplying each $f$ satisfying (3.3) by functions

$$
\hat{\chi}_{\varepsilon}(z)=\int e^{\langle z, t\rangle} \chi_{\varepsilon}(t) d \lambda(t),
$$

where

$$
\chi_{\varepsilon}(t)=\frac{1}{\varepsilon^{2 n}} \chi(t / \varepsilon)
$$

and $0 \leqq \chi(t)$ is a $C^{\infty}$ function on $R^{n}$ with compact support such that $\int \chi(t) d \lambda(t)=1$, to obtain a function $F_{\varepsilon}(z)=f(z) \hat{\chi}_{\varepsilon}(z)$ which, for $\varepsilon$ small, still satisfies (3.1) and which is in $L^{2}$ on the imaginary subspace of $\mathbb{C}^{n}$. As $\varepsilon \rightarrow 0^{+}, F_{\varepsilon} \rightarrow f$ in $\mathfrak{X}(\phi)$, and the Paley-Weiner Theorem implies that $F_{\varepsilon}$ is of the form in (3.3). Lastly, it is easy to prove that each $f$ of the form (3.3) is a limit in $\mathfrak{2}(\phi)$ of linear combinations of the $\exp (\langle z, t\rangle)$. This completes the proof.

In view of Theorem 5, to study when the polynomials are dense in $\mathfrak{A}(\phi)$ for $\phi$ as above, it suffices to find when the $\exp (\langle z, t\rangle)$ can be approximated by polynomials. The answer to this question is given by the following theorem, which is in fact equivalent to the DenjoyCarleman theorem on quasi-analytic functions.

THEOREM 6. If $\phi(z)=v(r)+u(x)$ satisfies (i)-(v) of this section, then the functions in $E(u)$ can be approximated by polynomials in $\mathfrak{U}^{p}(\dot{\phi}), 1 \leqq p \leqq+\infty$, if and only if

$$
\int_{0}^{+\infty} \frac{v(\tau r)}{1+\tau^{2}} d \tau=+\infty
$$

for every $r=\left(r_{1}, \cdots, r_{n}\right)$. Consequently, the polynomials are dense in $\mathfrak{Y}^{p}(\phi), 1 \leqq p \leqq+\infty$ if and only if (3.4) holds.

Proof. We first show that if (3.4) fails, then the polynomials cannot be dense in $\mathfrak{U}^{p}(\dot{\phi})$. We shall prove this only for the case $p=$ $+\infty$, as the other cases may be easily deduced from this by arguments 
analogous to those used previously to pass from one value of $p$ to all other values of $p$. Thus, assume that for some $r \neq(0, \cdots, 0)$

$$
\int_{0}^{+\infty} \frac{v(s r)}{1+s^{2}} d s<+\infty \text {. }
$$

It is no loss of generality to suppose that $\exp (\langle z, r\rangle) \in \mathfrak{P}^{\infty}(\dot{\phi})$, since if not, we can replace $r$ by $\varepsilon r$ for some small $\varepsilon>0$. We claim that $\exp (\langle z, r\rangle)$ cannot be approximated by polynomials in $\mathfrak{U}^{\infty}(\dot{\phi})$. For, assume that $P_{n}$ is a sequence of polynomials converging to $\exp (\langle z, r\rangle)$ in $\mathfrak{U}^{\infty}(\phi)$. Then for some constant $C>0$,

$$
\left|P_{n}(z)\right| \leqq C e^{v(|z|)-u(x)} \text {. }
$$

In particular, if $\zeta=a+i b$ is a complex number and $F_{n}(\zeta)=P_{n}(r \zeta)$, then $F_{n}$ is a polynomial in $\zeta$ and

$$
\log \left|F_{n}(i b)\right| \leqq v(|b| r)+u(0)+\log C .
$$

For the polynomials $F_{n}$, we have the well-known majorization given by Poisson's formula,

$$
\log \left|F_{n}(a+i b)\right| \leqq \frac{1}{\pi} \int_{-\infty}^{+\infty} \frac{a}{a^{2}+(b-\tau)^{2}} \log \left|F_{n}(i \tau)\right| d \tau,(a>0) .
$$

In particular, for $a>0$, we have

$$
\log \left|F_{n}(a)\right| \leqq u(0)+\log C+a \int_{-\infty}^{+\infty} \frac{v(\tau r)}{a^{2}+\tau^{2}} d \tau
$$

However,

$$
\int_{-\infty}^{+\infty} \frac{v(\tau r)}{a^{2}+\tau^{2}} d \tau \rightarrow 0 \quad \text { as } \quad a \rightarrow+\infty
$$

if (3.4) fails. Therefore, since the $P_{n}$ are assumed to converge to $\exp (\langle z, r\rangle)$ in $\mathfrak{U}^{\infty}(\phi)$, we have for $\left.a\right\rangle 0$

$$
a\langle r, r\rangle=\lim _{n \rightarrow++\infty} \log \left|F_{n}(a)\right| \leqq o(a)+\text { Const },
$$

which implies $\langle r, r\rangle=0$ and therefore $r=0$, a contradiction. Thus, $\exp \langle z, r\rangle$ cannot be a limit of polynomials in $\mathfrak{Y}^{\infty}(\dot{\phi})$.

We now prove the sufficiency of (3.4), again only for $p=\infty$, as the other cases are similar. To simplify the notation and computations, let us assume we are in the case of two complex variables. Define $C$ to be the open set in $R^{2}$ on which $u^{*}\left(t_{1}, t_{2}\right)$ is finite. Then let $F$ be the set of all $\left(t_{1}, t_{2}\right)$ in $\bigcirc$ for which $P\left(z_{1}, z_{2}\right) e^{t_{1} z_{1}+t_{2} z_{2}}$ is a limit of polynomials in $\mathfrak{P}^{\infty}(\phi)$ for all polynomials $P$. It is easy to check that $F$ is a (relatively) closed subset of $O$. Therefore, if $F$ is also an open 
subset we have $F=\mathcal{O}$, since $\mathcal{O}$ is convex, hence connected, and this is what we had to prove.

To prove that $F$ is open, we first prove the following apparently weaker assertion.

$\left(^{*}\right)$ If $u^{*}\left(t_{1}, t_{2}\right)<+\infty$ for $\left|t_{1}\right|<2 \varepsilon,\left|t_{2}\right|<2 \varepsilon$ then $F$ contains

$$
\left\{\left(t_{1}, t_{2}\right):\left|t_{1}\right|<\varepsilon,\left|t_{2}\right|<\varepsilon\right\} \text {. }
$$

The proof of $\left(^{*}\right)$ is based on the Denjoy-Carleman theorem. Let $L$ be a continuous linear functional on $\mathfrak{X}^{\infty}(\phi)$ which annihilates all polynomials. By the Hahn-Banach theorem, it is enough to prove that

$$
L\left(z_{1}^{n} z_{2}^{m} e^{t_{1} z_{1}+t_{2} z_{2}}\right)=0 \text { for }\left|t_{1}\right|<\varepsilon,\left|t_{2}\right|<\varepsilon .
$$

Define for such $t_{1}, t_{2}$,

$$
f\left(t_{1}, t_{2}\right)=L\left(e^{t_{1} z_{1}+t_{2} z_{2}}\right) .
$$

Then $f$ is infinitely differentiable and

$$
\frac{\partial^{n+m}}{\partial t_{1}^{n} \partial t_{2}^{m}} f\left(t_{1}, t_{2}\right)=L\left(z_{1}^{n} z_{2}^{m} e^{t_{1} z_{1}+t_{2} z_{2}}\right)
$$

so it suffices to prove that $f\left(t_{1}, t_{2}\right)=0$. Now

$$
\left|\frac{\partial^{n}}{\partial t_{1}^{n}} f\left(t_{1}, 0\right)\right|=\left|L\left(z_{1}^{n} e^{t_{1} z_{1}}\right)\right| \leqq C\left\|z_{1}^{n} e^{t_{1} z_{1}}\right\|
$$

since $L$ is continuous. However,

$$
\left|z_{1}^{n} e^{t_{1} z_{1}}\right| \leqq e^{u^{*}\left(t_{1}, 0\right)} \sup _{r} r_{1}^{n} e^{-v\left(r_{1}, 0\right)}
$$

and the sequence of constants

$$
b_{n}=\sup _{r_{1}} r_{1}^{n} e^{-v\left(r_{1}, 0\right)}
$$

determine a quasi-analytic class since (3.4) holds with $r=(1,0)$ (see, e.g., [11]). Also,

$$
\frac{\partial^{n}}{\partial t_{1}^{n}} f(0,0)=0, n=0,1,2, \cdots,
$$

so $f\left(t_{1}, 0\right)=0,\left|t_{1}\right|<\varepsilon$.

Now we also assert that $\left(t_{1}, 0\right) \in F$. For, from the above argument with $v\left(r_{1}, 0\right)$ replaced by $\frac{1}{2} v\left(r_{1}, 0\right)$ and $u(x)$ replaced by $\varepsilon\left|x_{1}\right|$, it follows that there is a sequence of polynomials in $z_{1}$ which converges to

$$
e^{t_{1} z_{1}},\left|t_{1}\right|<\varepsilon,
$$

in the Banach space $\mathfrak{U}^{\infty}\left(\frac{1}{3} v\left(r_{1}, 0\right)+\varepsilon\left|x_{1}\right|\right)$ of entire functions of one 
variable. In particular, there are polynomials $P_{n}$ such that

$$
\left|P_{n}\left(z_{1}\right)-e^{t_{1} z_{1}}\right| \leqq \frac{1}{n} e^{1 / 2 v\left(r_{1}, 0\right)+\varepsilon\left|x_{1}\right|} .
$$

Then

$$
\begin{aligned}
& \left|z_{2}^{m} P_{n}\left(z_{1}\right)-z_{2}^{m} e^{t_{1} z}\right| \leqq \frac{r_{2}^{m}}{n} e^{1 / 2 v\left(r_{1}, 0\right)+\varepsilon\left|x_{1}\right|} \\
& \leqq \frac{\text { Const. }}{n} e^{1 / 2 v\left(0, r_{2}\right)+1 / 2 v\left(r_{1}, 0\right)+u\left(x_{1}, x_{2}\right)} \\
& \leqq \frac{\text { Const. }}{n} e^{v\left(r_{1}, r_{2}\right)+u\left(x_{1}, x_{2}\right)}
\end{aligned}
$$

since $v\left(r_{1}, r_{2}\right)$ is nondecreasing in each variable. Therefore,

$$
P_{n}\left(z_{1}\right) z_{2}^{m} \rightarrow z_{2}^{m} e^{t_{1} z_{1}}
$$

and consequently

$$
\frac{\partial^{m}}{\partial t_{2}^{m}} f\left(t_{1}, 0\right)=L\left(z_{2}^{m} e^{t_{1} z_{1}}\right)=0,\left|t_{1}\right|<\varepsilon .
$$

Then also

$$
\frac{\partial^{n+m}}{\partial t_{1}^{n} \partial t_{2}^{m}} f\left(t_{1}, 0\right)=0
$$

so $\left(t_{1}, 0\right) \in F$ as asserted.

We now proceed as earlier and apply the Denjoy-Carleman Theorem. Thus

$$
\begin{aligned}
& \left|\frac{\partial^{m}}{\partial t_{2}^{m}} f\left(t_{1}, t_{2}\right)\right| \leqq C\left\|z_{2}^{m} e^{t_{1} z_{1}+t_{2} z_{2}}\right\| \\
& \left.\leqq C e^{u^{*}\left(t_{1}, t_{2}\right.}\right) \sup _{r}\left(r_{2}^{m} e^{-v\left(0, r_{2}\right)}\right)
\end{aligned}
$$

and hence for fixed $t_{1}, f\left(t_{1}, t_{2}\right)=g\left(t_{2}\right)$ belongs to a quasi-analytic class. As verified above

$$
\frac{\partial^{m}}{\partial t_{2}^{m}} f\left(t_{1}, 0\right)=0, m=0,1,2, \cdots
$$

so $f\left(t_{1}, t_{2}\right)=0,\left|t_{1}\right|<\varepsilon,\left|t_{2}\right|<\varepsilon$, which completes the proof of $\left(^{*}\right)$.

It remains to prove that $F$ is open. Let $\left(t_{1}, t_{2}\right) \in F$. Choose $\varepsilon>0$ so small that $\left(t_{1}+s_{1}, t_{2}+s_{2}\right) \in \mathcal{O}$ for $\left|s_{1}\right|<2 \varepsilon,\left|s_{2}\right|<2 \varepsilon$. Then let $\widetilde{u}\left(x_{1}, x_{2}\right)=u\left(x_{1}, x_{2}\right)-t_{1} x_{1}-t_{2} x_{2}$. We have that $\widetilde{u}^{*}\left(s_{1}, s_{2}\right)<+\infty$ for $\left|s_{1}\right|<2 \varepsilon,\left|s_{2}\right|<2 \varepsilon$. Applying $\left(^{*}\right)$ with the weight function $v\left(r_{1}, r_{2}\right)+$ $\widetilde{u}\left(x_{1}, x_{2}\right)$, we obtain the existence of polynomials $P_{n}$ converging to $e^{s_{1} z_{1}+s_{2} z_{2}}$ in $\mathfrak{U}^{\infty}\left(v\left(r_{1}, r_{2}\right)+\widetilde{u}\left(x_{1}, x_{2}\right)\right)$. But the norm of 


$$
P_{n} e^{t_{1} z_{1}+t_{2} z_{2}}-e^{\left(t_{1}+s_{1}\right) z_{1}+\left(t_{2}+s_{2}\right) z_{2}}
$$

in the space with weight $v\left(r_{1}, r_{2}\right)+u\left(x_{1}, x_{2}\right)$ is equal to the norm of $P_{n}-e^{s_{1} z_{1}+s_{2} z_{2}}$ in the space with weight $v\left(r_{1}, r_{2}\right)+\widetilde{u}\left(x_{1}, x_{2}\right)$. Therefore $e^{\left(t_{1}+s_{1}\right) z_{1}+\left(t_{2}+s_{2}\right) z_{2}}$ is a limit of polynomials in $\mathfrak{H}^{\infty}(\phi)$ for all $\left|s_{1}\right|<\varepsilon,\left|s_{2}\right|<\varepsilon$. It is easy to deduce from this that

$$
z_{1}^{n} z_{2}^{m} e^{\left(t_{1}+s_{1}\right) z_{1}+\left(t_{2}+s_{2}\right) z_{2}}
$$

is also a limit of polynomials so that $\left(t_{1}+s_{1}, t_{2}+s_{2}\right) \in F$. This completes the proof.

4. Examples. We conclude with some simple examples. Consider the weight functions $\phi(z)=|x|^{s}+|y|^{t}$, where $z_{j}=x_{j}+i y_{j},|x|=\sum\left|x_{j}\right|$, and $|y|=\sum\left|y_{j}\right|$. Then

(A) The polynomials are dense in $\mathfrak{U}^{p}(\phi)$ if $s \geqq 1, t \geqq 1$, or if $s<1$ and $t<1$.

(B) The polynomials are not dense in $\mathfrak{U}^{p}(\phi)$ if $s \geqq 1, t<1$ or $s<1$, $t \geqq 1$.

The first part of (A) is a consequence of Theorem 2, and (B) follows from Theorem 6, which shows that the exponential functions cannot be approximated by polynomials even in the space with a larger weight function $|x|^{s}+r^{t}$. The other part of (A) is a consequence of a Phragmen-Lindelöf theorem. For, if, say $s \leqq t<1$, then it follows from a Phragmen-Lindelöf theorem that each $f \in \mathfrak{U}^{p}(\phi)$ satisfies

$$
|f(z)| \leqq C \exp \left(\frac{1}{p}\left(\left|z_{1}\right|+\cdots+\left|z_{n}\right|\right)^{s}\right) .
$$

However, $\left|z_{1}\right|+\cdots+\left|z_{n}\right|^{s} \leqq|x|^{s}+|y|^{s} \leqq|x|^{s}+|y|^{t}$ for $|y| \geqq 1$. From these estimates, it follows easily that the Taylor series for $f(\theta z)$ converges to $f(\theta z)$ in $\mathfrak{A}^{v}(\phi)$ for each $\theta<1$. It is also clear that $f(\theta z) \rightarrow$ $f(z)$ in $\mathfrak{A}^{p}(\phi)$ as $\theta \rightarrow 1$, so the polynomials are dense, as asserted.

Lastly, we give an example where the topology is much weaker than a norm topology but in which polynomial approximation still fails. It is easy to construct such examples using Theorem 6. For instance, let

$$
\begin{aligned}
\mathscr{F}= & \left\{f(x+i y) \text { entire: }\|f\|_{\varepsilon}=\sup \left|f(z) \exp \left(-|z|^{1 / 2+\varepsilon}-|x|^{2+\varepsilon}\right)\right|\right. \\
& \left.<+\infty \text { for each } 0<\varepsilon<\frac{1}{2}\right\} .
\end{aligned}
$$

Then $\mathscr{F}$ is a Fréchet space with the topology determined by the seminorms, \|\|$_{\varepsilon}$, and it follows from Theorem 6 that $e^{z}$ cannot be approximated in $\mathscr{F}$ by polynomials. 
A result similar to Theorem 1 has been proved by J. P. Ferrier (Approximation with bounds of holomorphic functions of several complex variables, to appear in Ann. Inst. Fourier, Grenoble). In addition, two independent proofs of Theorem 2 have been given in the dissertations of D. Wohlgelenter (Yeshiva University) and J. Metzger (University of Michigan).

\section{REFERENCES}

1. R. P. Boas, Entire Functions, Academic Press, 1954.

2. L. Ehrenpreis, Analytically uniform spaces and some applications, Trans. Amer. Math. Soc. 101 (1961), 52-74.

3. _ Fourier Analysis in Several complex Variables, Interscience, (1970).

4. W. Fenchel, Convex Cones, Sets, and Functions, Princeton University Notes (Department of Mathematics), 1953.

5. E. Hewitt and K. Stromberg, Real and Abstract Analysis, Springer-Verlag, 1965.

6. L. Hörmander, $L^{2}$ estimates and existence theorems for the op operator, Acta. Math. 113 (1965), 89-152.

7. An Introduction to Complex Analysis in Several Variables, Van Nostrand, 1966.

8. Generators for some rings of analytic functions, Bull. Amer. Math. Soc. 73 (1967), 943-949.

9. L. Hormander and J. Wermer, Uniform approximation on compact sets in $\mathbb{E}^{n}$, Math. Scand. 23 (1968).

10. C. O. Kiselman, Existence of entire functions of one variable with prescribed indicator, Arkiv For Mathematik, 7 (1968), 505-508.

11. S. Mandelbrojt, Series de Fourier et Classes Quasi-Analytiques de Fonctions, Gauthier-Villars, Paris, 1935.

12. A. Martineau, Sur les fonctionelles analytiques et la transformation de FourierBorel, J. D'Analyse Math. 11 (1963), 1-164.

13. R. Nirenberg and R. O. Wells, Holomorphic approximation on real submanifolds of a complex manifold, Bull. Amer. Math. Soc. 73 (1967), 378-381.

14. B. A. Taylor, "Some locally convex spaces of entire functions" Entire Functions and Related Parts of Analysis, Proceedings of Symposia in Pure and Applied Mathematics, Vol. 11, Amer. Math. Soc.

15. __ Some analytically uniform spaces of in finitely differentiable functions (to appear in Comm. Pure and Appl. Math.).

Received April 23, 1969. This paper represents results obtained at the University of Michigan and the Courant Institute of Mathematical Sciences, New York University. The author gratefully acknowledges support from the National Science Foundation and the Sloan Foundation.

UNIVERSITY OF MichigaN 



\section{PACIFIC JOURNAL OF MATHEMATICS}

\section{EDITORS}

H. SAMELson

Stanford University

Stanford, California 94305

R. R. PHelPS

University of Washington

Seattle, Washington 98105
J. Dugundu

Department of Mathematics

University of Southern Californle

Los Angeles, California 9.0007

RICHARD ARENS

University of California

Los Angeles, California 9.0024

\section{ASSOCIATE EDITORS}
E. F. BECKENBACH
B. H. NeumanN
F. WOLE
K. Yoshida

\section{SUPPORTING INSTITUTIONS}

UNIVERSITY OF BRITISH COLUMBIA

CALIFORNIA INSTITUTE OF TECHNOLOGY

UNIVERSITY OF CALIFORNIA

MONTANA STATE UNIVERSITY

UNIVERSITY OF NEVADA

NEW MEXICO STATE UNIVERSITY

OREGON STATE UNIVERSITY

UNIVERSITY OF OREGON

OSAKA UNIVERSITY

UNIVERSITY OF SOUTHERN CALIFORNIA

\author{
STANFORD UNIVERSITY \\ UNIVERSITY OF TOKYO \\ UNIVERSITY OF UTAH \\ WASHINGTON STATE UNIVERSITY \\ UNIVERSITY OF WASHINGTON \\ AMERICAN MATHEMATICAL SOCIETY \\ CHEVRON RESEARCH CORPORATION \\ NAVAL WEAPONS CENTER
}

The Supporting Institutions listed above contribute to the cost of publication of this Journal, but they are not owners or publishers and have no responsibility for its content or policies.

Mathematical papers intended for publication in the Pacific Journal of Mathematics should be in typed form or offset-reproduced, (not dittoed), double spaced with large margins. Underline Greek letters in red, German in green, and script in blue. The first paragraph or two must be capable of being used separately as a synopsis of the entire paper. The editorial "we" must not be used in the synopsis, and items of the bibliography should not be cited there unless absolutely necessary, in which case they must be identified by author and Journal, rather than by item number. Manuscripts, in duplicate if possible, may be sent to any one of the four editors. Please classify according to the scheme of Math. Rev. Index to Vol. 39. All other communications to the editors should be addressed to the managing editor, Richard Arens, University of California, Los Angeles, California, 90024.

50 reprints are provided free for each article; additional copies may be obtained at cost in multiples of 50 .

The Pacific Journal of Mathematics is published monthly. Effective with Volume 16 the price per volume (3 numbers) is $\$ 8.00$; single issues, $\$ 3.00$. Special price for current issues to individual faculty members of supporting institutions and to individual members of the American Mathematical Society: $\$ 4.00$ per volume; single issues $\$ 1.50$. Back numbers are available.

Subscriptions, orders for back numbers, and changes of address should be sent to Pacific Journal of Mathematics, 103 Highland Boulevard, Berkeley, California, 94708.

PUBLISHED BY PACIFIC JOURNAL OF MATHEMATICS, A NON-PROFIT CORPORATION

Printed at Kokusai Bunken Insatsusha (International Academic Printing Co., Ltd.), 7-17, Fujimi 2-chome, Chiyoda-ku, Tokyo, Japan. 


\section{Pacific Journal of Mathematics}

\section{Vol. 36, No. 2 December, 1971}

George E. Andrews, On a partition problem of H. L. Alder ............ 279

Thomas Craig Brown, An interesting combinatorial method in the theory of locally finite semigroups .......................... 285

Yuen-Kwok Chan, A constructive proof of Sard's theorem ............. 291

Charles Vernon Coffman, Spectral theory of monotone Hammerstein

operators...................................... 303

Edward Dewey Davis, Regular sequences and minimal bases .......... 323

Israel (Yitzchak) Nathan Herstein and Lance W. Small, Regular elements in

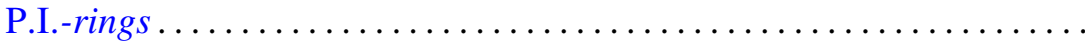

Marcel Herzog, Intersections of nilpotent Hall subgroups ..............

W. N. Hudson, Volterra transformations of the Wiener measure on the space

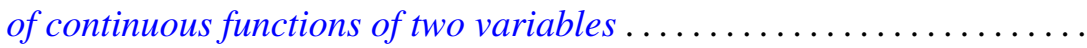

J. H. V. Hunt, An n-arc theorem for Peano spaces ................ 351

Arnold Joseph Insel, A decomposition theorem for topological group

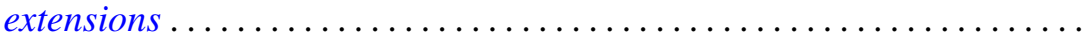

Caulton Lee Irwin, Inverting operators for singular boundary value

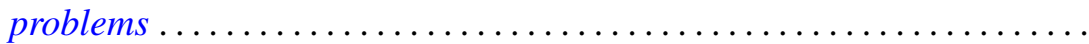

Abraham A. Klein, Matrix rings of finite degree of nilpotency ............ 387

Wei-Eihn Kuan, On the hyperplane section through a rational point of an algebraic variety...

John Hathway Lindsey, II, On a six-dimensional projective representation of $\mathrm{PSU}_{4}(3)$

Jorge Martinez, Approximation by archimedean lattice cones ...

J. F. McClendon, On stable fiber space obstructions .........

Mitsuru Nakai and Leo Sario, Behavior of Green lines at the Kuramochi boundary of a Riemann surface ....................

Donald Steven Passman, Linear identities in group rings. I. .

Donald Steven Passman, Linear identities in group rings. II ...

David S. Promislow, The Kakutani theorem for tensor products of

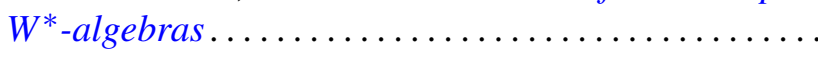

Richard Lewis Roth, On the conjugating representation of a finite group

Bert Alan Taylor, On weighted polynomial approximation of entire functions...

William Charles Waterhouse, Divisor classes in pseudo Galois

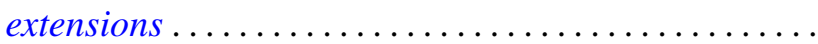

Chi Song Wong, Subadditive functions ...

Ta-Sun $\mathrm{Wu}$, A note on the minimality of certain bitransformation groups 